\title{
Seguimiento de un fibroma ameloblástico con transformación maligna a fibrosarcoma ameloblástico.
}

Galindo-Obando $\mathrm{U}^{1}$, Delgado-Azañero $\mathrm{W}^{2}$, Calderón-Ubaqui $\mathrm{V}^{2}$, Beltrán-Silva $\mathrm{J}^{2}$, Huamaní-Parra $\mathrm{J}^{2}$, Paniura-Rodriguez $\mathrm{D}^{3}$, Anchayhua-Espinoza $\mathrm{M}^{3}$. Seguimiento de un fibroma ameloblástico con transformación maligna a fibrosarcoma ameloblástico. Rev Estomatol Herediana. 2012; 22(1):26-30.

\section{RESUMEN}

La transformación maligna de un tumor odontogénico es poco común y puede desarrollarse a partir de un tumor odontogénico benigno. Se presenta el caso de una paciente de sexo femenino de 19 años de edad, con el seguimiento secuencial de las radiografías y tomografía computarizada de una lesión inicialmente diagnosticada como Ameloblastoma, que posterior al tratamiento quirúrgico se establece como Fibroma ameloblastico y al cabo de seis años sufre una transformación maligna determinado con un diagnostico histológico a Fibrosarcoma ameloblastico.

El objetivo del presente reporte es exponer un caso que llego al Servicio de Medicina, Cirugía y Patología Oral de la Universidad Peruana Cayetano Heredia donde se recopilo los datos de la historia médica con los estudios de imágenes.

PALABRAS CLAVE: Fibroma ameloblastico, Fibrosarcoma ameloblastico, transformación maligna.

Ameloblastic fibroma with a malignant transformation to ameloblastic fibrosarcoma.

ABSTRACT

Malignant transformation of an odontogenic tumor is rare and can develop from a benign odontogenic tumor. We report the case of a female patient of 19 years old, with tracking sequential radiographs and CT scan of a lesion initially diagnosed as ameloblastoma, after surgical treatment that is established as ameloblastic fibroma, six years after malignant transformation occurs with a histologic diagnosis given to ameloblastic fibrosarcoma. The objective of this report is to present this case to be referred to the Department of Medicine, Surgery and Oral Pathology, Peruvian University Cayetano Heredia where collected data on medical history with imaging studies.

KEY WORDS: Fibrosarcoma, mandibular neoplasms, odontogenic tumors.

\section{Introducción}

El fibroma ameloblástico (FA) es un tumor benigno odontogénico mixto (epitelial y mesenquimal) de rara aparición. Constituye el $2 \%$ de todos los tumores odontogénicos, sin predilección de sexo. Se presenta mayormente entre los 10 a 20 años de edad con un promedio de 12 años. Su localización es más frecuente en la mandíbula, en sectores posteriores, asociándose a veces a un diente incluido. Está compuesto por tejido conectivo con apariencia de pulpa dental y componente epitelial parecido a la lámina dental (1-3). Las características radiográficas del FA se presentan como una lesión radiolucida multilocular o unilocular de límites definidos con borde esclerótico. La mayoría de las recidivas se atribuyen a resecciones incompletas $(1,4,5)$. Se han descrito casos de transformación maligna de FA en fibrosarcoma ameloblástico (FSA) $(1,3,4)$.

El FSA según la Organización Mundial de la Salud (OMS) es un tumor odontogénico con un epitelio benigno y componentes ectomesenquimales malignos es considerado como el homologo maligno del fibroma ameloblástico (FA) con ICD-O 9330/3. El FA es una lesión de los maxilares poco común y se origina de los restos de epitelio odontogénico $(2,3,6)$. Se ha descrito que el $44 \%$ de los FA sufren transformación maligna $(1,3,4)$. Carlos y colaboradores (7) encontraron solamente 62 casos publicados de FSA en la literatura hasta el año 2003 con datos de 59,6 \% en varones y $37.4 \%$ en mujeres, el $79 \%$ se presento en la mandíbula y el $21 \%$ en el maxilar superior. De acuerdo con los datos de la literatura el FA

\section{Ursula Galindo Obando' Wilson Delgado Azañero² Víctor Calderón Ubaqui ${ }^{2}$ Jorge Beltrán Silva ${ }^{2}$ Jaime Huamaní Parra² Dithel Paniura Rodriguez ${ }^{3}$ Miguel Anchayhua Espinoza ${ }^{3}$}

Cirujano Dentista, Especialista en Radiología Oral y Maxilofacial.

2Docente del departamento de Cirugía Bucomaxilofacial, Facultad de Estomatología Roberto Beltran - Universidad Peruana Cayetano Heredia

${ }^{3}$ Cirujano Dentista, Especialista en Cirugía Oral y Maxilofacial

\section{Correspondencia}

Ursula Liseth Galindo Obando

Av. Villarán 1107 - Dpto. 501, Lima 34. Perú.

Teléfono: 511995644650

E-mail: uleys@hotmail.com tiene mayor potencial biológico de agresividad en comparación con el de otros tipos de tumores odontogénicos benignos. Esta condición se basa fundamentalmente en su capacidad de recidiva $(3,7)$.

El FSA es un tumor odontogénico con un epitelio benigno $\mathrm{y}$ componentes ectomesenquimales malignos. Es considerado como el homólogo maligno de un fibroma ameloblástico. Conocido también con el nombre de Sarcoma ameloblástico, se presenta en un rango de edad de 3-89 años, con un promedio de 27,5 años $(1,4,6,8)$. Mosqueda et al. reportan que aproximadamente un tercio de los FSA tienen como etiología la transformación maligna de un FA preexistente, el resto de casos estudiados surgen como lesiones sarcomatosas desde su inicio (6). La localización más 
frecuente del tumor es la mandíbula (78\%), seguido de la maxila (20\%). La región posterior es el sitio mas afectado. Solo ha sido publicado un caso de FSA periférico $(1,4,6,8)$.

Presenta características clínicas y radiográficas como tumefacción, dolor o parestesia, radiolucencia intraósea expansiva con bordes mal definidos (4).

La histopatología muestra un tejido epitelial benigno con componente conectivo maligno. El epitelio esta compuesto por cordones en brotes células epiteliales poligonales pequeñas mezcladas con islotes, estroma de tejido conectivo hipercelular. Los tumores recurrentes tienden a mostrar mayor celularidad estromal e índice mitótico elevado. El comportamiento biológico de los FSA data de una neoplasia local altamente agresiva con potencial extremadamente bajo para una metástasis a distancia. De 64 casos reportados, solo uno reporta metástasis a los nódulos mediastinales y al hígado (8).

El objetivo de este reporte fue exponer los signos radiográficos mostrados en la recopilación de un caso de recurrencia y posterior transformación maligna de un fibroma ameloblástico (tumor odontogénico benigno) a un fibrosarcoma ameloblástico (tumor odontogénico maligno).

\section{Reporte de caso}

Paciente de sexo femenino de 19 años de edad, refiere que seis años atrás, le realizan una exodoncia de una pieza dentaria molar del maxilar inferior lado derecho por presentar movilidad dentaria; presentando luego una tumoración mandibular en la misma zona por lo que acude al Instituto Nacional de Enfermedades Neoplásicas donde es diagnosticada como ameloblastoma, recibiendo tratamiento quirúrgico conservador. Al realizar los controles radiográficos en el primer año post quirúrgico se evidenció persistencia de la lesión observándose una radiolucidez homogénea y corticalizada que se interpretó como recidiva de ameloblastoma; sin embargo la paciente desistió de realizarse tratamiento.

Luego de cinco años de su último control acude al Servicio de Medicina, Cirugía y Patología Oral de la Clínica Dental Docente de la Facultad de Estomatología de la Universidad Peruana Cayetano Heredia, donde se hizo una reevaluación clínica, radiográfica y se recabó la lámina histológica inicial, siendo analizada y dando como resultado fibroma ameloblástico. En la evaluación clínica, se observó asimetría facial por aumento de volumen en la región mentoniana del lado derecho (Figura 1). Al realizar el examen intraoral se apreció un tumor ubicado en toda la extensión del reborde alveolar derecho, áreas de solución de continuidad, parasinfisis y sínfisis mandibular, borramiento del fondo de surco, consistencia firme y doloroso a la palpación, ausencia de piezas dentarias anteroinferiores y posteriores del cuarto cuadrante (Figura 2).

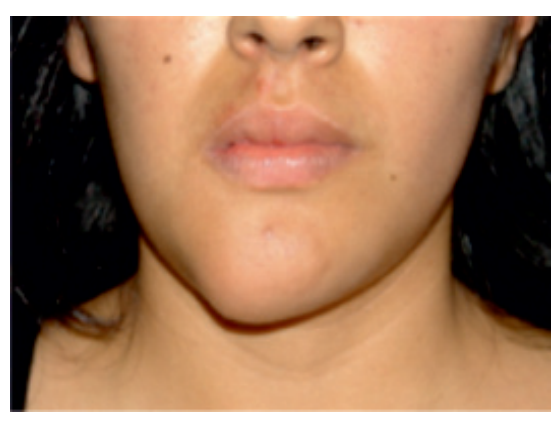

Figura 1. Aumento de volumen en la región mentoniana del lado derecho.

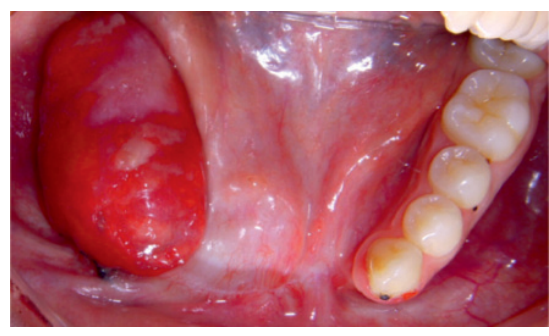

Fig. 2 Tumoración intraoral en reborde alveolar del IV cuadrante. Áreas de solución de continuidad, borramiento de fondo de surco, ausencia de piezas anteroinferiores y piezas posteriores del IV cuadrante.

El estudio imaginológico consistió en solicitar las radiografías pasadas, tomar una nueva radiografía panorámica y tomografía computarizada de macizo facial. La radiografía panorámica inicial del año 2002 mostraba una lesión radiolúcida multilocular en cuerpo mandibular derecho, de límites definidos, parcialmente corticalizados, patrón pseudoloculado y con extensión desde la pieza 43 a pieza 47 . Imagen de densidad blanda en zona de pieza 45, reabsorción radicular de pieza 4.6, ensanchamiento del espacio del ligamento periodontal en pieza 44, desplazamiento de pieza 43 y divergencia radicular. Características radiográficas compatibles con Ameloblastoma (Figura 3).

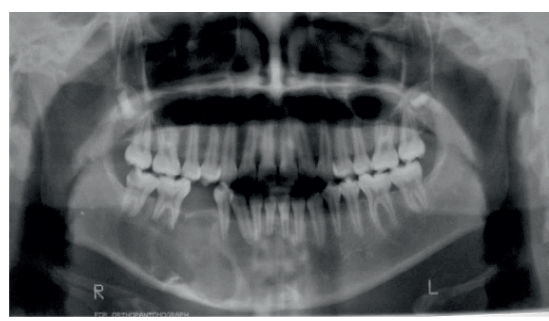

Figura 3. Radiografía panorámica inicial año 2002. Lesión radiolucida multilocular en cuerpo mandibular derecho.

En la radiografía panorámica de control del año 2003 se distingue una imagen radiolucida de límites definidos, parcialmente corticalizada, festoneada y ubicada en cuerpo mandibular del lado derecho que indica recidiva de la lesión (Fig 4). 


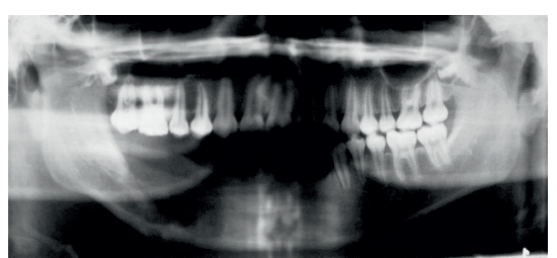

Figura 4. Radiografía panorámica año 2003. Imagen radiolúcida compatible con recidiva de lesión

A la evaluación de la radiografía panorámica de control en el año 2004 se advierte la persistencia de la lesión radiolucida multiloculada con incremento en su tamaño, perforación de la basal mandibular (Figura 5).

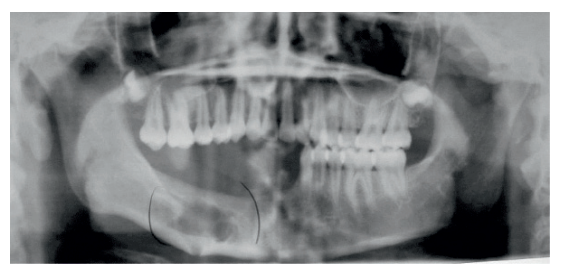

Figura 5. Radiografía panorámica año 2004. Persistencia de lesión radiolúcida.

La evaluación imaginológica actual nos muestra en la radiografía panorámica una lesión radiolúcida multilocular, localizada en cuerpo mandibular, que cruza la línea media y se instaura al lado contralateral, con aspecto de panal de abejas, límites parcialmente definidos $\mathrm{y}$ bordes parcialmente corticalizados. A nivel de sínfisis se extiende del proceso alveolar a la basal mandibular produciendo erosión, adelgazamiento, expansión, deformación y perforación de tablas óseas (Figura 6).

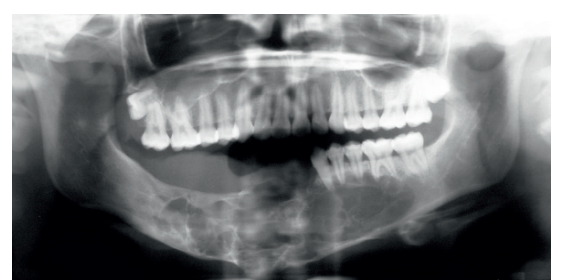

Fig. 6 Radiografía panorámica actual, se observa lesión radiolucida multilocular, que produce erosión, adelgazamiento, expansión, deformación y perforación de tablas óseas.
En la radiografía oclusal se observa la lesión multilocular en cuerpo mandibular, produciendo adelgazamiento, expansión, deformación y perforación de tablas óseas vestibular y lingual con presencia de fino trabeculado (Fig. 7).

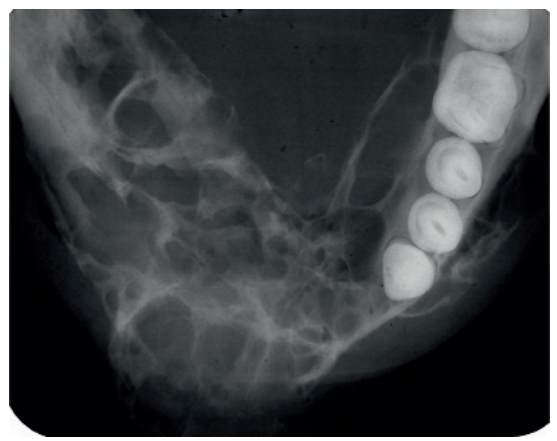

Fig. 7 Radiografía oclusal, lesión multilocular, adelgazamiento, expansión, deformación y perforación de tablas óseas vestibular y lingual, presencia de fino trabeculado.

Las radiografías periapicales mostraron reabsorción radicular de tipo multiplanar, "filo de cuchillo", ensanchamiento del espacio del ligamento periodontal de piezas adyacentes a la lesión (Figura 8).
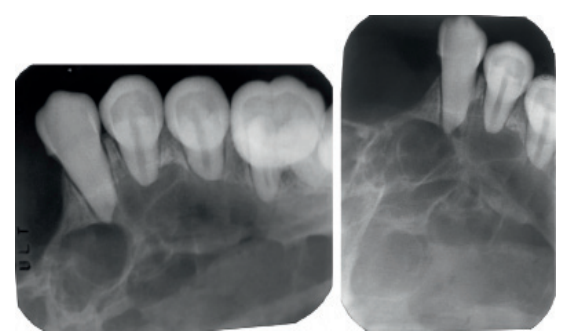

Figura 8. Radiografías periapicales, reabsorción radicular, ensanchamiento del espacio del ligamento periodontal de piezas adyacentes a la lesión.

En la tomografía computarizada espiral multicorte de macizo facial en ventana de tejidos óseos y blandos con aplicación de sustancia de contraste se puede observar una lesión neoformativa osteolítica mandibular parcialmente corticalizada con áreas de necrosis, septos, hiper captación periférica y central, herniación a tejidos blandos (Figuras 9, 10, 11 y 12).

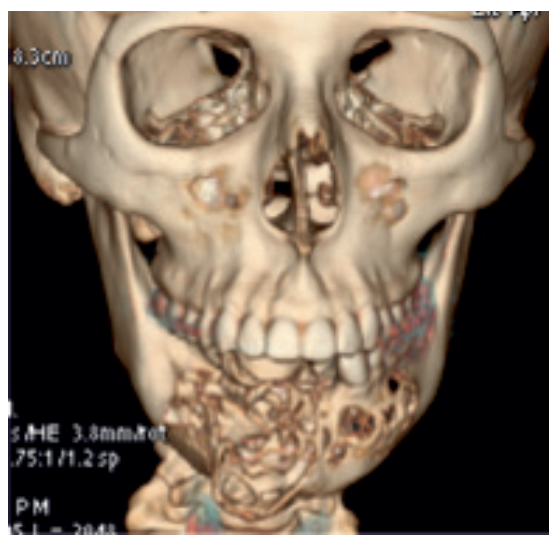

Figura 9. Tomografía Computarizada en reconstrucción $3 D$, cortes axial, coronal y sagital se observa: Lesión osteolítica mandibular con herniación a tejidos blandos.

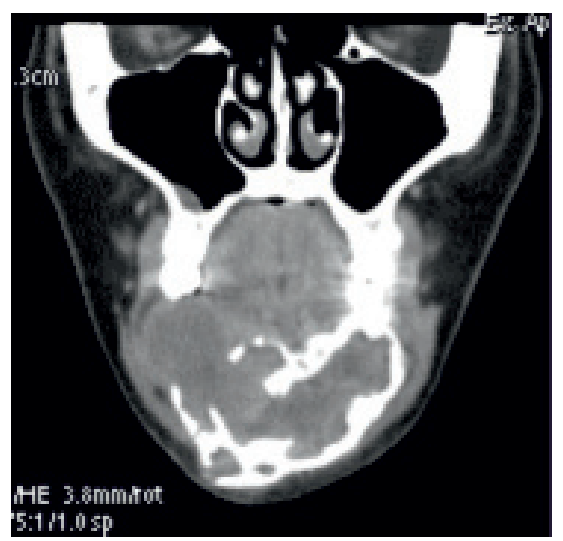

Figura 10. Tomografía Computarizada en reconstrucción $3 D$, cortes axial, coronal y sagital se observa: Lesión osteolítica mandibular con herniación a tejidos blandos

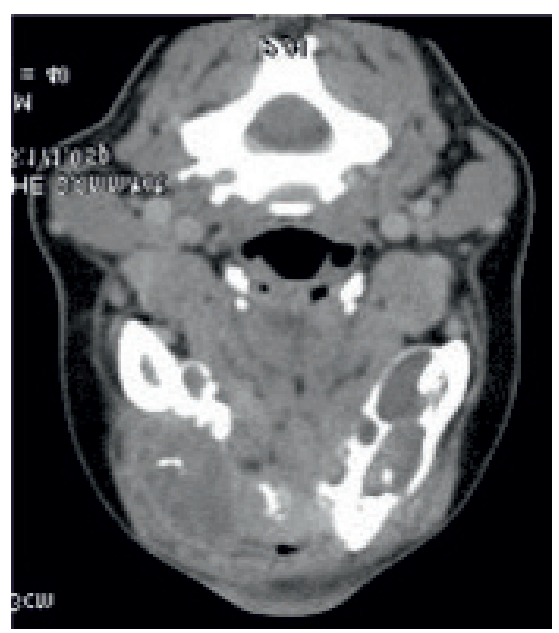

Figura 11. Tomografía Computarizada en reconstrucción $3 D$, cortes axial, coronal y sagital se observa: Lesión osteolítica mandibular con herniación a tejidos blandos 


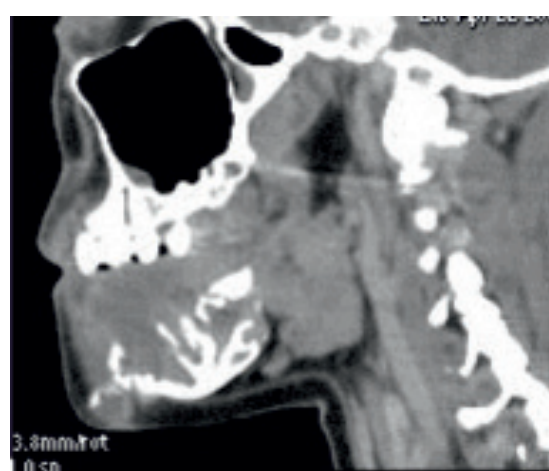

Figura 12. Tomografía Computarizada en reconstrucción 3D, cortes axial, coronal y sagital se observa: Lesión osteolítica mandibular con herniación a tejidos blandos.

A la evaluación imaginológica se encuentran características de agresividad y probable malignidad por perforación de tablas óseas e invasión a tejidos blandos.

Entre los diagnósticos presuntivos radiográficos se consideró una lesión tumoral odontogénica recidivante agresiva; sin embargo, la existencia de características imaginológicas descritas de lesión maligna nos lleva a considerar el descarte de transformación maligna.

El estudio anatomopatológico de la pieza quirúrgica mostró epitelio odontogénico tipo ameloblastos formando islas y rodeado de células fusiformes con hipercromatismo y núcleos grandes. Hipercelularidad, atipia celular, pleomorfismo celular, hipercromatismo, pleomorfismo nuclear, mitosis atípica. Siendo el diagnóstico definitivo Fibrosarcoma ameloblástico de bajo grado (Figura 13).

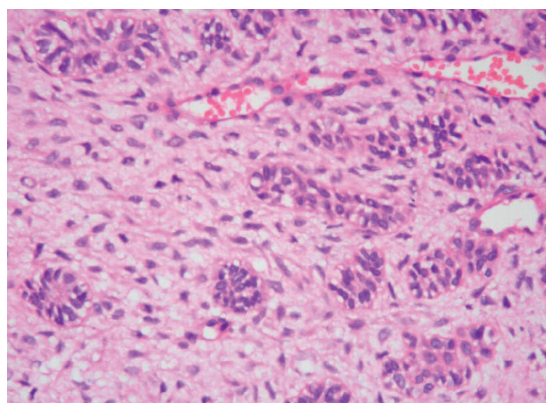

Fig.13 Epitelio odontogénico tipo ameloblastos, hipercelularidad y atipia (bajo grado, atipia celular.
El tratamiento fue resección quirúrgica segmentaria con colocación de una placa de reconstrucción e injerto óseo córtico-esponjoso de la cresta ilíaca, luego se realizó una radiografía panorámica y radiografía postero anterior para el control radiográfico post quirúrgico (Figu$\operatorname{ras} 14,15,16$ y 17$)$.

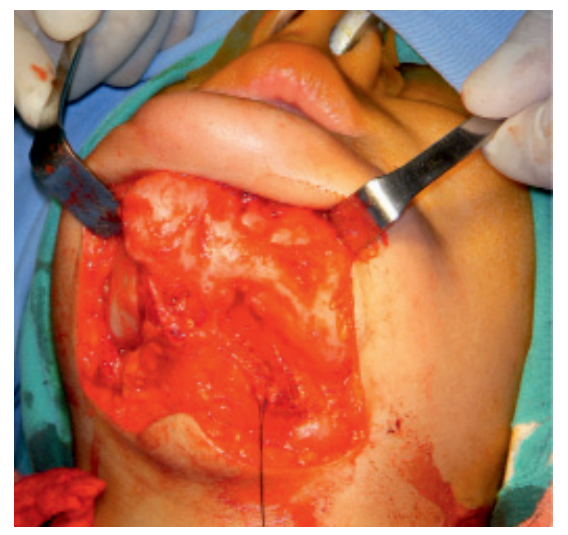

Fig.14 Resección quirúrgica.

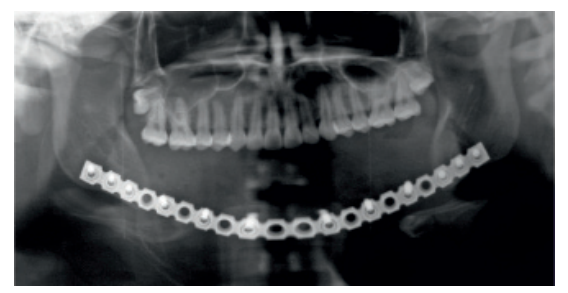

Fig.15 Colocación de una placa de reconstrucción.

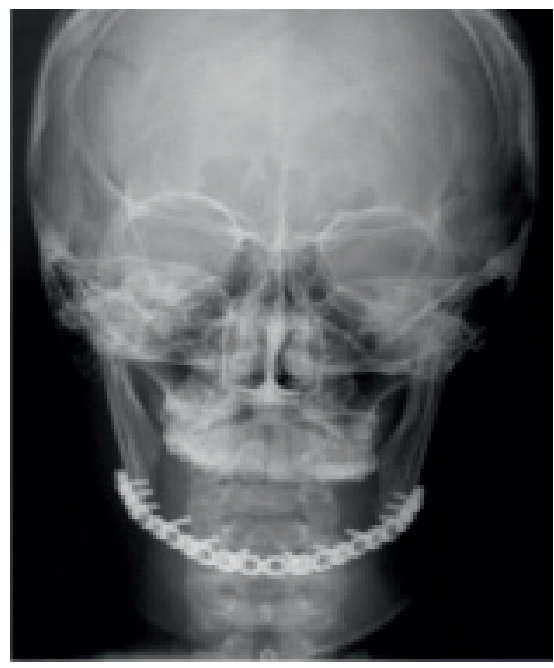

Fig.16 Injerto óseo córtico-esponjoso de la cresta ilíaca.

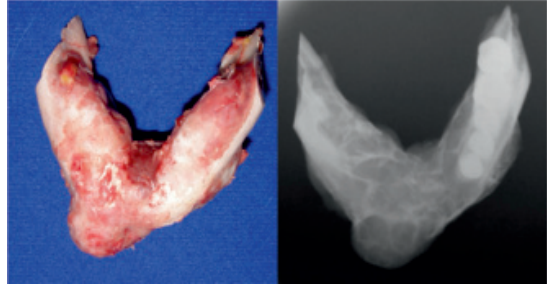

Fig. 17 Macroscopía y radiografía de la pieza quirúrgica.

\section{Discusión}

Los tumores odontogénicos malignos (TOMs) constituyen un grupo heterogéneo de lesiones que afectan a los maxilares y a la región bucal, muchos de estos son generalmente considerados como los homólogos de los tumores odontogénicos benignos, son clasificados en carcinomas odontogénicos y sarcomas odontogénicos. Son entidades raras y su etiología es desconocida y han demostrado su desarrollo a partir de los tumores odontogénicos benignos preexistentes. El diagnóstico oportuno de estas lesiones es complejo por su baja frecuencia, escasa información sobre sus características clínicas e histológicas y la similitud de estas neoplasias con los tumores odontogénicos benignos que trae como consecuencia un diagnóstico errado como lesión benigna.

Mosquera et al. reportan una baja frecuencia de TOMs dando como explicación el hecho de que la mayoría de estas lesiones se originan a nivel central y en etapas iniciales cursan de forma asintomática, en fases mas avanzadas producen expansión cortical, dolor, desplazamiento y movilidad dental simulando otras lesiones de mayor prevalencia como los tumores odontogénicos benignos, siendo estos datos similares al presente caso y por lo tanto la dificultad para su identificación y tratamiento (6).

Según Eversole, la mayoría de 
los casos reportados son lesiones primarias, existen lesiones que se han producido por transformación maligna, particularmente después de haber sido sometidas a múltiples intervenciones quirúrgicas (9). Nuestro caso tuvo antecedente de tumor odontogénico benigno diagnosticado cinco años atrás y tratado quirúrgicamente en forma incompleta. La histología actual mostró signos de malignidad. Muller et al. relatan que más de una tercera parte de los casos de FSA se han originado a partir de un fibroma ameloblástico pre existente (10). En nuestro caso los estudios de las zonas de lesión demuestran la existencia de un fibroma ameloblástico desde su inicio.

El presente caso constituye un FA en una paciente de sexo femenino de presentación inicial a los 13 años de edad, localizado en la mandíbula en el sector posterior de acuerdo con la frecuencia del FA. La tumefacción fue el signo clínico más notable. La lesión fue diagnosticada como ameloblastoma guiando el tratamiento quirúrgico conservador, la reevaluación histológica posterior a la recidiva concluye que se trataba de un fibroma ameloblástico, presentándose entonces signos radiográficos de recidiva en los controles. La evaluación tomográfica presentó características de malignidad considerando como diagnóstico diferencial recidiva de Fibroma ameloblastico a descartar transformación maligna en fibrosarcoma ameloblastico. Establecido el diagnóstico definitivo como fibrosarcoma ameloblástico, se procedió a la resección radical y la indicación de seguimiento posterior de por lo menos dos años como indica la literatura (10-12).

Es necesario realizar un estudio por imágenes que involucre a la radiología convencional y la tomografía computarizada para tener una mejor evidencia de posibilidad de neoplasia maligna. Las características imaginológicas, pueden incluir una gama de lesiones como tumores odontogénicos benignos agresivos, pero son precisas a la hora de evaluar las señales de malignidad como perforación de tablas óseas, efecto de masa, instauración de la lesión en el lado contralateral cruzando la línea media, invasión a tejidos blandos, siendo característicos en casos de agresividad y transformación maligna. El examen anatomopatológico es necesario para el diagnostico de la lesión y el planeamiento quirúrgico por el alto potencial de recidiva y malignidad de este tipo de lesiones. El seguimiento por imágenes de una neoplasia benigna es de vital importancia para descartar recidivas en el tiempo o posibilidad de transformación maligna.

\section{Referencias Bibliográficas}

1. DeLair D, Bejarano PA, Peleg M, El-Mofty SK. Ameloblastic carcinosarcoma of the mandible arising in ameloblastic fibroma: a case report and review of the literature. Oral Surg Oral Med Oral Pathol Oral Radiol Endod. 2007; 103(4):516-20.

2. Barnes L, Eveson J, Reichart P, Sidransky D; World Health Organization classification of tumours. Pathology and genetics of head and neck tumours. Lyon: IARC Press;2005.

3. Chen Y, Wang JM, Li TJ. Ameloblastic fibroma: a review of published studies with special reference to its nature and biological behavior. Oral Oncol. 2007; 43(10):960-9.

4. Langlais R, Langland O, Nortjé C. Diagnostic imaging of the jaws. Baltimore: Williams \& Wilkins; 1995. p. 327-335.

5. Goaz P, White P. Radiología oral. 3ra Edición. Madrid: Mosby; 1995.

6. Mosqueda-Taylor A, MenesesGarcía A, Ruíz-Godoy-Rivera LM, Suárez-Roa ML, Luna-Ortiz K. Tumores odontogénicos malignos: Estudio retrospectivo $\mathrm{y}$ colaborativo de 7 casos. Med Oral. 2003; 8:110-21.

7. Bregni RC, Taylor AM, García AM. Ameloblastic fibrosarcoma of the mandible: report of two cases and review of the literature. J Oral Pathol Med. 2001; 30(5):316-20.

8. DeLair D, Bejarano PA, Peleg M, El-Mofty SK. Ameloblastic carcinosarcoma of the mandible arising in ameloblastic fibroma: a case report and review of the literature. Oral Surg Oral Med Oral Pathol Oral Radiol Endod. 2007; 103(4):516-20.

9. Eversole LR. Malignant epithelial odontogenic tumors. Semin Diagn Pathol. 1999; 16(4):31724.

10. Muller S, Parker DC, Kapadia SB, Budnick SD, Barnes EL. Ameloblastic fibrosarcoma of the jaws: A clinicopathologic and DNA analysis of five cases and review of the literature with discussion of its relationship to ameloblastic fibroma. Oral Surg Oral Med Oral Pathol Oral Radiol Endod. 1995; 79(4):469-77.

11. Hayashi Y, Tohnai I, Ueda M, Nagasaka T. Sarcomatous overgrowth in recurrent ameloblastic fibrosarcoma. Oral Oncol. 1999; 35(3):346-8.

12. Chomette G, Auriol M, Guilbert F, Delcourt A. Ameloblastic fibrosarcoma of the jaws--report of three cases. Clinico-pathologic, histoenzymological and ultrastructural study. Pathol Res Pract. 1983; 178(1):40-7. 